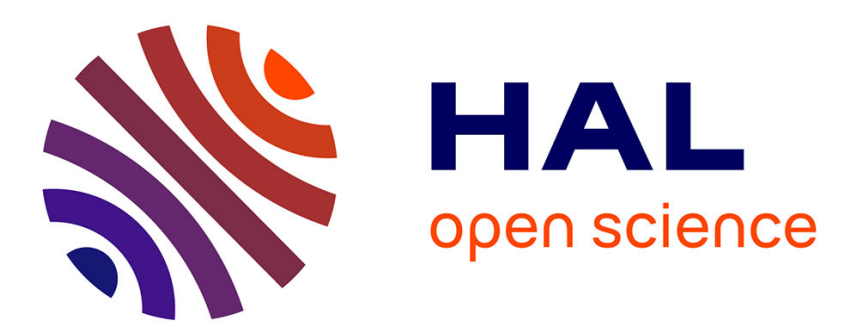

\title{
Constrained Control of Uncertain, Time-varying Linear Discrete-Time Systems Subject to Bounded Disturbances
}

Hoaï-Nam Nguyen, Sorin Olaru, Per-Olof Gutman, Morten Hovd

\section{- To cite this version:}

Hoaï-Nam Nguyen, Sorin Olaru, Per-Olof Gutman, Morten Hovd. Constrained Control of Uncertain, Time-varying Linear Discrete-Time Systems Subject to Bounded Disturbances. IEEE Transactions on Automatic Control, 2015, 60 (3), pp.831-836. 10.1109/TAC.2014.2346872 . hal-01257659

\section{HAL Id: hal-01257659 \\ https://hal.science/hal-01257659}

Submitted on 19 Jan 2016

HAL is a multi-disciplinary open access archive for the deposit and dissemination of scientific research documents, whether they are published or not. The documents may come from teaching and research institutions in France or abroad, or from public or private research centers.
L'archive ouverte pluridisciplinaire HAL, est destinée au dépôt et à la diffusion de documents scientifiques de niveau recherche, publiés ou non, émanant des établissements d'enseignement et de recherche français ou étrangers, des laboratoires publics ou privés. 


\title{
Constrained control of uncertain, time-varying linear discrete-time systems subject to bounded disturbances
}

\author{
H.-N. Nguyen ${ }^{\dagger}$, S. Olaru ${ }^{\ddagger}$, P.-O. Gutman ${ }^{\dagger}$, M. Hovd ${ }^{\dagger \ddagger}$
}

\begin{abstract}
The aim of this paper is twofold. In the first part, robust invariance for ellipsoidal sets with respect to uncertain and/or time-varying linear discrete-time systems with bounded additive disturbances is revisited. We provide an extension of an existing invariance condition. In the second part a novel robust interpolation based control design involving several local unconstrained robust optimal controls is proposed. At each time instant a quadratic programming problem is solved on-line. Proofs of recursive feasibility and input-to-state stability are given.
\end{abstract}

\section{INTRODUCTION}

In this paper we consider robust control of uncertain and/or time-varying linear discrete-time systems affected by bounded additive disturbances. Input, state and disturbance constraints are taken into account in the control design. This control problem has been tackled with e.g. invariant set methods [1], or model predictive control (MPC) [2].

In the MPC context, one approach is to formulate a minmax optimization problem [3] which is NP-hard. In [4] tube-MPC is proposed for nominal systems with bounded disturbances. The design is complicated and it is non-trivial to extend it to uncertain and/or time-varying plants.

Here interpolating robust constrained control is considered. On-line interpolation is not a new concept, see e.g [5] where interpolation between several asymptotically stabilizing feedback controllers is performed by minimizing an upper bound on the infinite horizon objective function. However, these results do not allow for priority among the interpolating control laws.

We provide, firstly, a necessary and sufficient condition for the positive invariance of an ellipsoid with respect to uncertain and/or time-varying systems with bounded additive disturbances. This invariance is an extension of a result in [6]. Secondly, a robust control method for constrained uncertain and/or time-varying systems subject to bounded disturbances is introduced, based on interpolation between $r$ local unconstrained robust optimal controllers. It has three main features:

a) Recursive feasibility and input to state stability (ISS) are guaranteed for all feasible initial conditions. b) At each time instant, the solution of a quadratic programming (QP) problem of dimension $(r-1)(n+1)$ is required, where $n$ is the state dimension. c) With a block diagonal choice of the

$\dagger$ Faculty of Civil and Environmental Engineering, Technion - Israel Institute of Technology, Haifa 32000, Israel \{nam, peo\}@technion.ac.i

$\ddagger$ SUPELEC Systems Sciences (E3S) - Automatic Control Department, Gif sur Yvette, France sorin.olaruesupelec.fr

$\dagger \ddagger$ Department of Engineering Cybernetics, Norwegian University of Science and Technology morten.hovdeitk.ntnu.no cost function matrix, the minimal robust positively invariant set for the performance controller is shown to be an attractor.

The paper is partially based on the conference contribution [7] where some further examples are found.

Notation: $\mathbb{I}$ and $\mathbf{0}$ denote the identity and zeros matrices, respectively, of appropriate dimensions. Whenever time is omitted, a variable $x$ stands for $x(k)$ for some $k \in \mathbb{N}$.

\section{PROBLEM FORMULATION}

Consider the following uncertain and/or time-varying linear discrete-time system,

$$
x(k+1)=A(k) x(k)+B(k) u(k)+E d(k)
$$

where $x(k) \in \mathbb{R}^{n}, u(k) \in \mathbb{R}^{m}$ and $d(k) \in \mathbb{R}^{d}$ are, respectively, the measured state vector, the control input and the unknown additive disturbance. The matrices $A(k) \in$ $\mathbb{R}^{n \times n}, B(k) \in \mathbb{R}^{n \times m}$ and $E \in \mathbb{R}^{n \times d} . A(k)$ and $B(k)$ satisfy

$$
A(k)=\sum_{i=1}^{s} \alpha_{i}(k) A_{i}, \quad B(k)=\sum_{i=1}^{s} \alpha_{i}(k) B_{i}
$$

where $\sum_{i=1}^{s} \alpha_{i}(k)=1, \alpha_{i}(k) \geq 0$ and $A_{i}, B_{i}$ are given. $x(k), u(k)$ and $d(k)$ are subject to the following polytopic constraints

$\left\{\begin{array}{l}x(k) \in X, X=\left\{x \in \mathbb{R}^{n}:\left|F_{j x} x\right| \leq 1\right\}, j=1, \ldots, n_{x} \\ u(k) \in U, U=\left\{u \in \mathbb{R}^{m}:\left|u_{j}\right| \leq u_{j \max }\right\}, j=1, \ldots, m \\ d(k) \in D, D=\left\{d \in \mathbb{R}^{d}:\left|F_{j d} d\right| \leq 1\right\}, j=1, \ldots, n_{d}\end{array}\right.$

where $F_{j x}$ and $F_{j d}$ are respectively, the $j-t h$ row vector of the matrices $F_{x} \in \mathbb{R}^{n_{x} \times n}$ and $F_{d} \in \mathbb{R}^{n_{d} \times d}, u_{\text {jmax }}$ is the $j-t h$ component of the vector $u_{\max } . F_{x}, F_{d}$ and $u_{\max }$ are assumed to be constant with $u_{\max }>0$ such that the origin is contained in the interior of $X, U$ and $D$.

A control law $u(k)=u(x(k))$ for (1) is to be designed such that the closed loop system is ISS w.r.t. $d(k)$ with the constraints (3) satisfied.

\section{PREliminaries: ISS STABILITy AND SET INVARIANCE}

Use will be made of $K$-functions, $K_{\infty}$-functions, $K L$-functions, ISS stability and ISS gain, and ISS Lyapunov functions as defined in [8].

Consider (1) with controller $u(k)=K x(k)$,

$$
x(k+1)=A_{c}(k) x(k)+E d(k)
$$

where,

$$
A_{c}(k)=A(k)+B(k) K=\sum_{i=1}^{s} \alpha_{i}(k)\left(A_{i}+B_{i} K\right) .
$$


Theorem 1 [8]: The system (4) is input-to-state stable if it admits an ISS-Lyapunov function.

Definition 1: (RPI) [1] A polyhedral set $\Omega \subseteq X$ is a robustly constraint-admissible positively invariant (RPI) set w.r.t. (4), (3) iff, $\forall x(k) \in \Omega$ and $\forall d(k) \in D$, it holds that $A_{c}(k) x(k)+E d(k) \in \Omega$ and $K x(k) \in U$.

$\Omega \subseteq X$ is the maximal RPI (MRPI) set for (4) and constraints (3) iff $\Omega$ is a RPI set and contains every RPI set. A non-empty MRPI set is unique, see [1], where a constructive procedure is given to compute it in polyhedral form, $\Omega=\left\{x \in \mathbb{R}^{n}: F_{o} x \leq g_{o}\right\}$.

Definition 2: (Invariant ellipsoid) An ellipsoid $\mathcal{E}(P)=$ $\left\{x \in \mathbb{R}^{n}: x^{T} P^{-1} x \leq 1\right\}$ is robustly positively invariant for (4) if $x(0) \in \mathcal{E}(P)$ implies $x(k) \in \mathcal{E}(P), \forall k \geq 1$.

\section{RoBUstness ANALYSIS AND CONTROLLER DESIGN FOR INVARIANT ELLIPSOIDS}

\section{A. Robustness analysis}

Consider (1) with $u(k)=K x(k)$ yielding the closed loop (4). How to compute $K \in \mathbb{R}^{m \times n}$ will be shown below. Using the vertex representation of $D$, whereby $d(k) \in D$, one can find the smallest outer ellipsoid $\mathcal{E}\left(P_{d}\right)=\{d \in$ $\left.\mathbb{R}^{d}: d^{T} P_{d} d \leq 1\right\}$, that contains $D$ [9]. Theorem 2 gives a necessary and sufficient condition for invariance of ellipsoids for system (4).

Theorem 2: $\mathcal{E}(P)$ is invariant for (4) iff the $P \in \mathbb{R}^{n \times n}$ satisfies the following LMI condition, for some scalar $0<$ $\tau<1, \forall i=1,2, \ldots, s$,

$$
\left[\begin{array}{ccc}
(1-\tau) P & \mathbf{0} & P\left(A_{i}+B_{i} K\right)^{T} \\
* & \tau P_{d} & E^{T} \\
* & * & P
\end{array}\right] \succeq 0,
$$

In order to prove the result we revisit the technique proposed in [6].

Proof: Denote $V(x)=x^{T} P^{-1} x$. For the invariance property of $\mathcal{E}(P)=\left\{x(k) \in \mathbb{R}^{n}: V(x(k)) \leq 1\right\}$, it is required that $V(x(k+1)) \leq 1, \forall x(k) \in \mathcal{E}(P)$, and $\forall d(k) \in \mathcal{E}\left(P_{d}\right)$, i.e. $\left(A_{c} x+E d\right)^{T} P^{-1}\left(A_{c} x+E d\right) \leq 1$, $\forall x, d$ such that $x^{T} P^{-1} x \leq 1$ and $d^{T} P_{d} d \leq 1$, or

$$
\left[\begin{array}{l}
x \\
d
\end{array}\right]^{T}\left[\begin{array}{cc}
A_{c}^{T} P^{-1} A_{c} & A_{c}^{T} P^{-1} E \\
E^{T} P^{-1} A_{c} & E^{T} P^{-1} E
\end{array}\right]\left[\begin{array}{l}
x \\
d
\end{array}\right] \leq 1
$$

such that

$$
\begin{aligned}
& {\left[\begin{array}{l}
x \\
d
\end{array}\right]^{T}\left[\begin{array}{cc}
P^{-1} & \mathbf{0} \\
\mathbf{0} & \mathbf{0}
\end{array}\right]\left[\begin{array}{l}
x \\
d
\end{array}\right] \leq 1,} \\
& {\left[\begin{array}{l}
x \\
d
\end{array}\right]^{T}\left[\begin{array}{cc}
\mathbf{0} & \mathbf{0} \\
\mathbf{0} & P_{d}
\end{array}\right]\left[\begin{array}{l}
x \\
d
\end{array}\right] \leq 1}
\end{aligned}
$$

Using the $S$-procedure [10] with two quadratic constraints, (7), (8) is equivalent to,

$$
\left[\begin{array}{cc}
A_{c}^{T} P^{-1} A_{c} & A_{c}^{T} P^{-1} E \\
E^{T} P^{-1} A_{c} & E^{T} P^{-1} E
\end{array}\right] \preceq\left[\begin{array}{cc}
\tau_{1} P^{-1} & \mathbf{0} \\
\mathbf{0} & \tau_{2} P_{d}
\end{array}\right]
$$

for some value of $\tau_{1}>0, \tau_{2}>0$, such that $\tau_{1}+\tau_{2}<1$.

It holds that $A_{c}^{T} P^{-1} A_{c} \succ 0$ and $E^{T} P^{-1} E \succ 0$, since $P^{-1} \succ 0$. Clearly, the least restrictive right hand side of
(9) is obtained by setting $\tau=\tau_{2}=1-\tau_{1}$. Hence (9) is equivalent to the LMI

$$
\left[\begin{array}{cc}
(1-\tau) P^{-1} & \mathbf{0} \\
\mathbf{0} & \tau P_{d}
\end{array}\right]-\left[\begin{array}{c}
A_{c}^{T} \\
E^{T}
\end{array}\right] P^{-1}\left[\begin{array}{ll}
A_{c} & E
\end{array}\right] \succeq 0
$$

Using the Schur complement, one obtains

$$
\left[\begin{array}{ccc}
(1-\tau) P^{-1} & \mathbf{0} & A_{c}^{T} \\
\mathbf{0} & \tau P_{d} & E^{T} \\
A_{c} & E & P
\end{array}\right] \succeq 0
$$

or equivalently, for some scalar $0<\tau<1$,

$$
\left[\begin{array}{ccc}
(1-\tau) P & \mathbf{0} & P A_{c}^{T} \\
\mathbf{0} & \tau P_{d} & E^{T} \\
A_{c} P & E & P
\end{array}\right] \succeq 0
$$

It follows from (5) that the left hand side of (10) is linear with respect to $\alpha_{i}(k)$. Hence one should verify (10) at the vertices of $\alpha_{i}(k)$, i.e. when $\alpha_{i}(k)=0$ or $\alpha_{i}(k)=1$. Therefore the LMI conditions to be satisfied are (6).

Remark: Theorem 2 extends the LMI condition in [6], where a similar condition was used to identify the minimal invariant ellipsoids for linear systems. The LMI (6) is a necessary and sufficient condition for ellipsoids to be invariant for uncertain and/or time-varying systems. In addition, (6) is applicable for generic ellipsoidal invariance, e.g. minimal, maximal, etc.

\section{B. Robust optimal design}

There are several conflicting objectives for designing a controller for system (1) with constraints (3). Usually, one would like to have an invariant ellipsoid with a large domain of attraction. It is well known [9] that by using the LMI technique, one can determine the largest invariant ellipsoid $\mathcal{E}(P)$ with respect to the inclusion of some reference direction defined by $x_{p}$, meaning that the set $\mathcal{E}(P)$ will include the point $\theta x_{p}$, where $\theta$ is a scaling factor. Indeed, $\theta x_{p} \in \mathcal{E}(P)$ implies that $\theta^{2} x_{p}^{T} P^{-1} x_{p} \leq 1$ or by using the Schur complement,

$$
\left[\begin{array}{cc}
1 & \theta x_{p}^{T} \\
\theta x_{p} & P
\end{array}\right] \succeq 0
$$

Therefore, by using a linear feedback controller $u=K x$, an invariant ellipsoid $\mathcal{E}(P)$, that contains the largest extension in a certain direction defined by the reference point $x_{p}$, can be obtained by solving the following LMI problem,

$$
\max _{P, Y, \theta}\{\theta(\tau)\}
$$

subject to

- Invariance condition (6), which can be reformulated to be linear in $P$ and $Y$.

- Reference point inclusion (11).

- Constraint satisfaction [9].

$$
\begin{aligned}
& \text { On state: }\left[\begin{array}{cc}
1 & F_{j x} P \\
P F_{j x}^{T} & P
\end{array}\right] \succeq 0, \forall j=1,2, \ldots, n_{x} \\
& \text { On input: }\left[\begin{array}{cc}
u_{j \max }^{2} & Y_{j} \\
Y_{j}^{T} & P
\end{array}\right] \succeq 0, \forall j=1,2, \ldots, m
\end{aligned}
$$


where $Y=K P \in \mathbb{R}^{m \times n}, K_{j}$ is the $j-t h$ row of $K$ and $Y_{j}=K_{j} P$ is the $j-t h$ row of $Y$.

Note that when $\tau$ is fixed, the optimization problem (12) is an LMI problem, for which there nowadays exist several effective parsers and solvers, e.g. [11].

Remark: The reference points $x_{p}$ can be chosen according to the available information on the initial conditions. For example, if some possible initial conditions are known, we can choose a set of reference points, that contains all these initial conditions.

\section{INTERPOLATION BASED CONTROL}

Using the results in the previous section, a set of robust asymptotically stabilizing controllers $u=K_{t} x, t=$ $1,2, \ldots, r$ is obtained such that $A_{c t}(k)=A(k)+B(k) K_{t}$ are robustly asymptotically stable and the corresponding MRPI sets $\Omega_{t} \subseteq X$

$$
\Omega_{t}=\left\{x \in \mathbb{R}^{n}: F_{o t} x \leq g_{o t}\right\}, t=1,2, \ldots, r
$$

are non-empty. Define $\Omega$ as the convex hull of $\Omega_{t}, t=$ $1,2, \ldots, r$. It follows that $\Omega \subseteq X$, since $X$ is convex and $\Omega_{t} \subseteq X, \forall t=1,2, \ldots, r$. The first controller in this enumeration will play the role of a performance controller, while the remaining controllers will be used for enlarging the domain of attraction.

\section{A. Cost function determination}

We use a similar decomposition of the state vector as the one in [12]. Any $x(k) \in \Omega$ can be decomposed as,

$$
x(k)=\lambda_{1}(k) \widehat{x}_{1}(k)+\sum_{t=2}^{r} \lambda_{t}(k) \widehat{x}_{t}(k)
$$

where $\widehat{x}_{t}(k) \in \Omega_{t}, \forall t=1,2, \ldots, r$ are decomposition variables and will be treated as decision variables, $\lambda_{t}(k)$ are interpolating coefficients that satisfy $\sum_{t=1}^{r} \lambda_{t}(k)=1$, $\lambda_{t}(k) \geq 0, \forall t=1,2, \ldots, r$. Equation (13) can be rewritten as,

$$
x(k)=v_{1}(k)+\sum_{t=2}^{r} v_{t}(k)
$$

with $v_{t}(k)=\lambda_{t}(k) \widehat{x}_{t}(k)$. Hence,

$$
v_{1}(k)=x(k)-\sum_{t=2}^{r} v_{t}(k)
$$

Since $\widehat{x}_{t} \in \Omega_{t}$, it follows that $v_{t} \in \lambda_{t} \Omega_{t}$, or in other words,

$$
F_{\text {ot }} v_{t} \leq \lambda_{t} g_{o t}, \quad t=1,2, \ldots, r
$$

Consider the following control law,

$$
\begin{aligned}
u(k) & =\lambda_{1}(k) K_{1} \widehat{x}_{1}(k)+\sum_{t=2}^{r} \lambda_{t}(k) K_{t} \widehat{x}_{t}(k) \\
& =K_{1} v_{1}(k)+\sum_{t=2}^{r} K_{t} v_{t}(k)
\end{aligned}
$$

where $K_{t} \widehat{x}_{t}(k)$ is the control law in $\Omega_{t}, \forall t=1,2, \ldots, r$.
Substituting (15) into (17), one gets

$$
\begin{aligned}
u(k) & =K_{1}\left(x(k)-\sum_{t=2}^{r} v_{t}(k)\right)+\sum_{t=2}^{r} K_{t} v_{t}(k) \\
& =K_{1} x(k)+\sum_{t=2}^{r}\left(K_{t}-K_{1}\right) v_{t}(k)
\end{aligned}
$$

Using (18), one obtains,

$x(k+1)=A_{c 1}(k) x(k)+B(k) \sum_{t=2}^{r}\left(K_{t}-K_{1}\right) v_{t}(k)+E d(k)$

where $A_{c 1}(k)=A(k)+B(k) K_{1}$.

Define $v_{t}(k+1), t=2,3, \ldots, r$ as

$$
v_{t}(k+1)=A_{c t}(k) v_{t}(k)+E d_{t}(k)
$$

with $A_{c t}(k)=A(k)+B(k) K_{t}, d_{t}(k)=\lambda_{t}(k) d(k), \forall t=$ $2,3, \ldots, r$.

Define also the vectors $z$ and $w$ as

$z=\left[\begin{array}{llll}x^{T} & v_{2}^{T} & \ldots & v_{r}^{T}\end{array}\right]^{T}, w=\left[\begin{array}{llll}d^{T} & d_{2}^{T} & \ldots & d_{r}^{T}\end{array}\right]^{T}$

Writing (19), (20) in a compact matrix form, one obtains

$$
z(k+1)=\Phi(k) z(k)+\Gamma w(k)
$$

where

$$
\begin{aligned}
& \Phi(k)=\left[\begin{array}{cccc}
A_{c 1}(k) & B(k)\left(K_{2}-K_{1}\right) & \ldots & B(k)\left(K_{r}-K_{1}\right) \\
\mathbf{0} & A_{c 2}(k) & \ldots & \mathbf{0} \\
\vdots & \vdots & \ddots & \vdots \\
\mathbf{0} & \mathbf{0} & \ldots & A_{c r}(k)
\end{array}\right], \\
& \Gamma=\left[\begin{array}{cccc}
E & \mathbf{0} & \ldots & \mathbf{0} \\
\mathbf{0} & E & \ldots & \mathbf{0} \\
\vdots & \vdots & \ddots & \vdots \\
\mathbf{0} & \mathbf{0} & \ldots & E
\end{array}\right]
\end{aligned}
$$

From (2), it is clear that $\Phi(k)$ can be expressed as a convex combination of $\Phi_{i}$,

$$
\Phi(k)=\sum_{i=1}^{s} \alpha_{i}(k) \Phi_{i}
$$
where $\sum_{i=1}^{s} \alpha_{i}(k)=1, \alpha_{i}(k) \geq 0$ and $\Phi_{i}$ is obtained from the
vertices $A_{i}, B_{i}$.

Consider the following quadratic function,

$$
V(z)=z^{T} \mathcal{P} z
$$

where the matrix $\mathcal{P} \in \mathbb{R}^{r n \times r n}, \mathcal{P} \succ 0$ is chosen to satisfy

$$
\begin{aligned}
& V(z(k+1))-V(z(k)) \leq \\
& \quad \leq-x(k)^{T} Q x(k)-u(k)^{T} R u(k)+\sigma w(k)^{T} w(k)
\end{aligned}
$$

with the weighting matrices $Q \in \mathbb{R}^{n \times n}, R \in \mathbb{R}^{m \times m}$ and $Q \succeq 0, R \succ 0, \sigma \geq 0$. 
Using (22), the left hand side of (25) can be written as,

$$
\begin{aligned}
& V(z(k+1))-V(z(k))= \\
& =(\Phi(k) z+\Gamma w)^{T} \mathcal{P}(\Phi(k) z+\Gamma w)-z^{T} \mathcal{P} z \\
& =\left[\begin{array}{ll}
z^{T} & w^{T}
\end{array}\right]\left[\begin{array}{c}
\Phi^{T}(k) \\
\Gamma^{T}
\end{array}\right] \mathcal{P}[\Phi(k) \quad \Gamma]\left[\begin{array}{c}
z \\
w
\end{array}\right]- \\
& -\left[\begin{array}{ll}
z^{T} & w^{T}
\end{array}\right]\left[\begin{array}{ll}
\mathcal{P} & \mathbf{0} \\
\mathbf{0} & \mathbf{0}
\end{array}\right]\left[\begin{array}{c}
z \\
w
\end{array}\right]
\end{aligned}
$$

and using (18), (21), the right hand side of (25) becomes,

$$
\begin{gathered}
-x(k)^{T} Q x(k)-u(k)^{T} R u(k)+\sigma w(k)^{T} w(k)= \\
=z(k)^{T}\left(-Q_{1}-R_{1}\right) z(k)+\sigma w(k)^{T} w(k) \\
=\left[\begin{array}{ll}
z^{T} & w^{T}
\end{array}\right]\left[\begin{array}{cc}
-Q_{1}-R_{1} & \mathbf{0} \\
\mathbf{0} & \sigma \mathbb{I}
\end{array}\right]\left[\begin{array}{c}
z \\
w
\end{array}\right]
\end{gathered}
$$

where

$$
\begin{aligned}
Q_{1} & =\left[\begin{array}{c}
\mathbb{I} \\
\mathbf{0} \\
\vdots \\
\mathbf{0}
\end{array}\right] Q\left[\begin{array}{cccc}
\mathbb{I} & \mathbf{0} & \ldots & \mathbf{0}
\end{array}\right], \\
R_{1} & =\left[\begin{array}{c}
K_{1}^{T} \\
\left.K_{2}-K_{1}\right)^{T} \\
\vdots \\
\left(K_{r}-K_{1}\right)^{T}
\end{array}\right] R\left[\begin{array}{llll}
K_{1} & \left(K_{2}-K_{1}\right) & \ldots & \left(K_{r}-K_{1}\right)
\end{array}\right]
\end{aligned}
$$

One obtains, from (25), (26), (27),

$$
\begin{aligned}
& {\left[\begin{array}{c}
\Phi^{T}(k) \\
\Gamma^{T}
\end{array}\right] \mathcal{P}\left[\begin{array}{ll}
\Phi(k) & \Gamma
\end{array}\right]-\left[\begin{array}{cc}
\mathcal{P} & \mathbf{0} \\
\mathbf{0} & \mathbf{0}
\end{array}\right] \preceq} \\
& \preceq\left[\begin{array}{cc}
-Q_{1}-R_{1} & \mathbf{0} \\
\mathbf{0} & \sigma \mathbb{I}
\end{array}\right]
\end{aligned}
$$

or equivalently

$$
\left[\begin{array}{cc}
\mathcal{P}-Q_{1}-R_{1} & \mathbf{0} \\
\mathbf{0} & \sigma \mathbb{I}
\end{array}\right]-\left[\begin{array}{c}
\Phi^{T}(k) \\
\Gamma^{T}
\end{array}\right] \mathcal{P}\left[\begin{array}{ll}
\Phi(k) & \Gamma
\end{array}\right] \succeq 0
$$

Using the Schur complement, (28) can be brought to

$$
\left[\begin{array}{ccc}
\mathcal{P}-Q_{1}-R_{1} & \mathbf{0} & \Phi^{T}(k) \mathcal{P} \\
* & \sigma \mathbb{I} & \Gamma^{T} \mathcal{P} \\
* & * & \mathcal{P}
\end{array}\right] \succeq 0
$$

Using (28), it is clear that (29) is feasible for $\sigma$ sufficiently large if $\Phi(k)$ is asymptotically stable.

The left hand side of (29) is linear with respect to $\alpha_{i}(k)$ in (23). Hence one should verify (29) at the vertices of $\alpha_{i}(k)$. Therefore the set of LMI conditions to be checked is as,

$$
\left[\begin{array}{ccc}
\mathcal{P}-Q_{1}-R_{1} & \mathbf{0} & \Phi_{i}^{T} \mathcal{P} \\
* & \sigma \mathbb{I} & \Gamma^{T} \mathcal{P} \\
* & * & \mathcal{P}
\end{array}\right] \succeq 0
$$

It is well known [8] that in the sense of the ISS gain having a smaller $\sigma$ is a desirable property. The smallest value of $\sigma$ can be found by solving the following LMI optimization problem,

$$
\min _{\mathcal{P}, \sigma}\{\sigma\} \text { subject to (30) }
$$

\section{B. Interpolation via quadratic programming}

Once the matrix $\mathcal{P}$ is computed as the solution of (31), it can be used in practice for real time control based on the resolution of a low complexity optimization problem. The resulting control law can be seen as a predictive control type of construction if the function (24) is interpreted as an upper bound for a receding horizon cost function.

At each time instant, for a given state $x$, minimize on-line the quadratic cost function,

$$
V_{1}\left(z, \lambda_{t}\right)=\min _{z, \lambda_{t}}\left\{z^{T} \mathcal{P} z+\sum_{t=2}^{r} \lambda_{t}^{2}\right\}
$$

subject to

$$
\left\{\begin{array}{l}
F_{o t} v_{t} \leq \lambda_{t} g_{o t}, \forall t=1,2, \ldots, r \\
\sum_{t=1}^{r} v_{t}=x \\
\sum_{t=1}^{r} \lambda_{t}=1, \lambda_{t} \geq 0
\end{array}\right.
$$

and implement as input the control action (18).

Denote the optimal solution of the QP problem (32) as $v_{t}^{o}(k)$ and $\lambda_{t}^{o}(k)$ and define $\widehat{x}_{t}^{o}$ such that $v_{t}^{o}(k)=\lambda_{t}^{o} \widehat{x}_{t}^{o}$, $t=1,2, \ldots, r$. Then the following theorem holds.

Theorem 3: The control law (18), (32) guarantees recursive feasibility and the closed loop system is ISS for all initial states $x(0) \in \Omega$.

Recursive feasibility proof: It has to be proved that $u(k) \in U$ and $x(k+1) \in \Omega, \forall x(k) \in \Omega$. Using (13), (17), (18) it follows that $x(k)$ and $u(k)$ can be expressed as,

$$
\left\{\begin{array}{l}
x(k)=\sum_{t=1}^{r} \lambda_{t}^{o}(k) \widehat{x}_{t}^{o}(k), \\
u(k)=K_{1} x(k)+\sum_{t=2}^{r}\left(K_{t}-K_{1}\right) v_{t}^{o}(k)=\sum_{t=1}^{r} \lambda_{t}^{o}(k) K_{i} \widehat{x}_{t}^{o}(k)
\end{array}\right.
$$

It thus holds that,

$$
\begin{aligned}
u(k) & =\sum_{t=1}^{r} \lambda_{t}^{o}(k) K_{i} \widehat{x}_{t}^{o}(k)=\sum_{t=1}^{r} \lambda_{t}^{o}(k) K_{t} \widehat{x}_{t}^{o}(k) \\
& \leq \sum_{t=1}^{r} \lambda_{t}^{o}(k) u_{\max }=u_{\max } \in U
\end{aligned}
$$

and,

$$
\begin{aligned}
x(k+1) & =A(k) x(k)+B(k) u(k)+E d(k) \\
& =\sum_{t=1}^{r} \lambda_{t}(k)\left\{\left(A(k)+B(k) K_{t}\right) \widehat{x}_{t}^{o}(k)+E d(k)\right\}
\end{aligned}
$$

Since $\left(A(k)+B(k) K_{t}\right) \widehat{x}_{t}^{o}(k)+E d(k) \in \Omega_{t} \subseteq \Omega$, it follows that $x(k+1) \in \Omega$.

ISS stability proof: From the feasibility proof, it is clear that if $v_{t}^{o}(k)$ and $\lambda_{t}^{o}(k)$ is the solution of (32) at time $k$, then

$$
v_{t}(k+1)=A_{c t}(k) v_{t}^{o}(k)+E d_{t}(k)
$$

and $\lambda_{t}(k+1)=\lambda_{t}^{o}(k)$ is a feasible solution of (32) at time $k+1$. Solving (32) at time $k+1$, one gets

$$
V_{1}\left(z^{o}(k+1), \lambda_{t}^{o}(k+1)\right) \leq V_{1}\left(z(k+1), \lambda_{t}(k)\right)
$$


and by using inequality (25), it follows that

$$
\begin{aligned}
& V_{1}\left(z^{o}(k+1), \lambda_{t}^{o}(k+1)\right)-V_{1}\left(z_{1}^{o}(k), \lambda_{t}^{o}(k)\right) \leq \\
& \quad \leq V_{1}\left(z(k+1), \lambda_{t}(k+1)\right)-V_{1}\left(z^{o}(k), \lambda_{t}^{o}(k)\right) \\
& \quad \leq-x(k)^{T} Q x(k)-u(k)^{T} R u(k)+\sigma w(k)^{T} w(k)
\end{aligned}
$$

Hence $V_{1}\left(z, \lambda_{t}\right)$ is an ISS Lyapunov function of (22). It follows that the closed loop system with the control law (18), (32) is ISS.

Remark: Matrix $\mathcal{P}$ can be chosen as,

$$
\mathcal{P}=\left[\begin{array}{cc}
\mathcal{S} & \mathbf{0} \\
\mathbf{0} & \mathcal{S}_{r}
\end{array}\right]
$$

where $\mathcal{S} \in \mathbb{R}^{n \times n}, \mathcal{S}_{r} \in \mathbb{R}^{(r-1) n \times(r-1) n}$. In this case, the cost function (32) can be written by

$$
V_{1}\left(z, \lambda_{t}\right)=x^{T} \mathcal{S} x+s_{r}^{T} \mathcal{S}_{r} s_{r}+\sum_{t=2}^{r} \lambda_{t}^{2}
$$

where $s_{r}=\left[\begin{array}{llll}v_{2}^{T} & v_{3}^{T} & \ldots & v_{r}^{T}\end{array}\right]^{T}$. Hence, for any $x(k) \in$ $\Omega_{1}$, the QP problem (32) has the trivial solution as $v_{t}^{o}=0$ and $\lambda_{t}^{o}=0, \forall t=2,3, \ldots, r$, and thus $v_{1}^{o}=x$ and $\lambda_{1}^{o}=1$. This choice of $\mathcal{P}$ is advantageous if controller $K_{1}$ is designed for performance, while the other controllers are used to enlarge the domain of attraction.

\section{EXAMPLE}

Consider the following uncertain linear discrete-time system,

$$
x(k+1)=A(k) x(k)+B(k) u(k)+d(k)
$$

where

$$
\begin{aligned}
& A(k)=\alpha(k) A_{1}+(1-\alpha(k)) A_{2}, \\
& B(k)=\alpha(k) B_{1}+(1-\alpha(k)) B_{2} \\
& A_{1}=\left[\begin{array}{rr}
1 & 0.1 \\
0 & 1 \\
1 & 0.2 \\
0 & 1
\end{array}\right], B_{1}=\left[\begin{array}{l}
0 \\
1 \\
0 \\
A_{2}=
\end{array}\right]
\end{aligned}
$$

$\alpha(k) \in[0,1]$ is a uncertain parameter. The constraints are: $|x|_{\infty} \leq 10,|u|_{\infty} \leq 1,|d|_{\infty} \leq 0.1$

Three controllers are chosen as

$$
\begin{aligned}
& K_{1}=\left[\begin{array}{ll}
-1.8112 & -0.8092
\end{array}\right], \\
& K_{2}=\left[\begin{array}{ll}
-0.0878 & -0.1176
\end{array}\right] \\
& K_{3}=\left[\begin{array}{ll}
-0.0979 & -0.0499
\end{array}\right]
\end{aligned}
$$

For $Q=\mathbb{I}$ and $R=1$, by solving (31) with $\mathcal{P}$ in the form (34), one obtains $\sigma=41150$ and

$$
\begin{aligned}
\mathcal{S} & =\left[\begin{array}{rrr}
76.2384 & 11.4260 \\
11.4260 & 3.6285
\end{array}\right], \\
\mathcal{S}_{3} & =\left[\begin{array}{rrrr}
2468.4 & 1.622 .9 & -144.2 & 105.2 \\
1622.9 & 4164.3 & -81.6 & -160.6 \\
-144.2 & -81.6 & 865.7 & 278.4 \\
105.2 & -160.6 & 278.4 & 967.8
\end{array}\right]
\end{aligned}
$$

Figure 1(a) shows the sets $\Omega_{1}, \Omega_{2}$ and $\Omega_{3}$ for the gains $K_{1}$, $K_{2}$ and $K_{3}$, respectively. Figure 1(b) presents state trajectories for different initial conditions and realizations of $\alpha(k)$ and $d(k)$. For the initial condition $x_{0}=\left[\begin{array}{ll}9.6145 & 1.1772\end{array}\right]^{T}$,

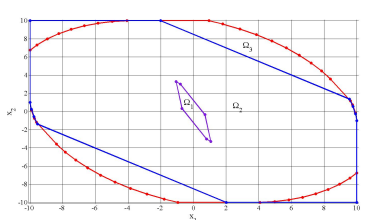

(a) Feasible sets

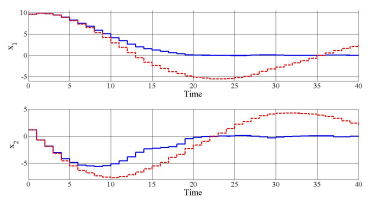

(c) State trajectories

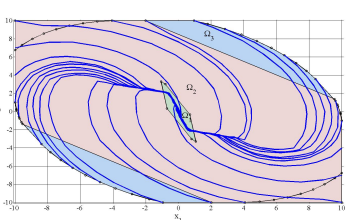

(b) State trajectories

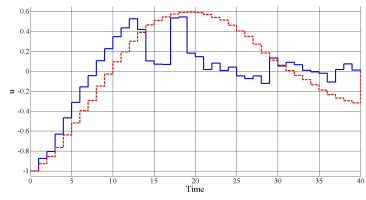

(d) Input trajectories
Fig. 1. (a-b) Feasible invariant sets and state trajectories. (c-d) State and input trajectories by our approach (solid blue) and by $u(k)=K_{3} x(k)$ (dashed red).

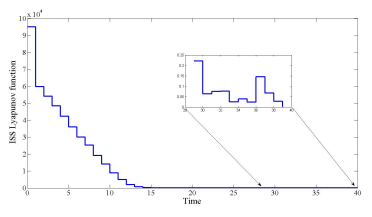

(a) ISS Lyapunov function

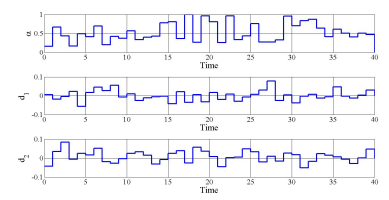

(c) $\alpha(k)$ and $d(k)$

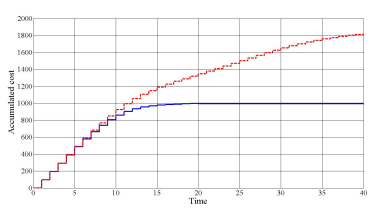

(b) Accumulated cost

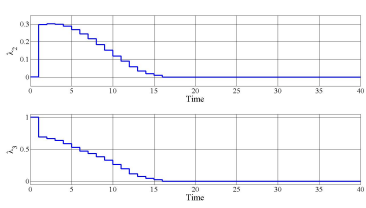

(d) $\lambda_{2}$ and $\lambda_{3}$
Fig. 2. (a-b) ISS Lyapunov function and its non-decreasing effect and the accumulated cost for our approach (solid blue) and for $u=K_{3} x$ (dashed red), (c-d) $\alpha(k)$ and $d(k)$ and $\lambda_{2}$ and $\lambda_{3}$.

Figures 1(c-d) show the state and input trajectories by our approach (solid blue) and by $u(k)=K_{3} x(k)$ (dashed red).

Figure 2(a) presents the ISS Lyapunov function. It is worth noticing than, when the state is near to the origin, $V_{1}\left(z_{1}\right)$ might be increasing at some instants as shown also in Figure 2(a). Figure 2(b) shows the accumulated cost $J(k)$, which is calculated as $J(0)=0$ and

$$
J(k+1)=J(k)+x(k)^{T} Q x(k)+u(k)^{T} R u(k)
$$

The realizations of $\alpha(k)$ and $d(k)=\left[\begin{array}{ll}d_{1}(k) & d_{2}(k)\end{array}\right]^{T}$ and the interpolating coefficients $\lambda_{2}$ and $\lambda_{3}$ are depicted in 2(c) and 2(d), respectively.

\section{CONCLUSION}

The present paper proposes two contributions: first we provide an extension of the robust invariance condition for ellipsoids with respect to uncertain and/or time-varying systems with bounded additive disturbances. Thereafter a novel interpolation scheme is introduced. The interpolation is done between several unconstrained robust controllers. Among them, one controller is used for the performance, while the others are used for enlarging the domain of 
attraction. The resulting control law guarantees recursive feasibility and ISS stability in the presence of constraints. A numerical example is presented to support the algorithms with illustrative simulations.

\section{REFERENCES}

[1] F. Blanchini and S. Miani, Set-theoretic methods in control. Springer, 2008.

[2] D. Mayne, J. Rawlings, C. Rao, and P. Scokaert, "Constrained model predictive control: Stability and optimality," Automatica, vol. 36, pp. 789-814, 2000.

[3] J. Löfberg, Minimax approaches to robust model predictive control. PhD Thesis, Linkoping Univ., 2003.

[4] S. Rakovic, B. Kouvaritakis, M. Cannon, C. Panos, and R. Findeisen, "Parameterized tube model predictive control," IEEE Transactions on Automatic Control, vol. 57, no. 11, pp. 2746-2761, 2012.

[5] M. Bacic, M. Cannon, Y. Lee, and B. Kouvaritakis, "General interpolation in mpc and its advantages," Automatic Control, IEEE Transactions on, vol. 48, no. 6, pp. 1092-1096, 2003

[6] S. Nazin, B. Polyak, and M. Topunov, "Rejection of bounded exogenous disturbances by the method of invariant ellipsoids," Automation and Remote Control, vol. 68, no. 3, pp. 467-486, 2007.

[7] H.-N. Nguyen, P.-O. Gutman, S. Olaru, and M. Hovd, "Robust optimization-based control of constrained linear discrete time systems with bounded disturbances," in Proceedings of the 5th IFAC Symposium on System Structure and Control, vol. 5, no. 1, 2013, pp. 917922.

[8] Z. Jiang and Y. Wang, "Input-to-state stability for discrete-time nonlinear systems," Automatica, vol. 37, no. 6, pp. 857-869.

[9] S. Boyd, L. El Ghaoui, E. Feron, and V. Balakrishnan, Linear matrix inequalities in system and control theory. Society for Industrial Mathematics, 1994, vol. 15.

[10] B. Polyak, "Convexity of quadratic transformations and its use in control and optimization," Journal of Optimization Theory and Applications, vol. 99, no. 3, pp. 553-583, 1998.

[11] M. Grant and S. Boyd, "Cvx: Matlab software for disciplined convex programming," Available http://cvxr.com/cvx/, 2008.

[12] J. Rossiter, B. Kouvaritakis, and M. Bacic, "Interpolation based computationally efficient predictive control," International Journal of Control, vol. 77, no. 3, pp. 290-301, 2004. 